\title{
Designing a Safer Interactive Healthcare System - The Impact of Authentic User Participation
}

\author{
Kathryn L. Went, Peter Gregor, and Ian W. Ricketts \\ School of Computing, University of Dundee, Dundee, \\ DD1 4HN, UK \\ \{kwent, pgregor, ricketts\}@computing.dundee.ac.uk
}

\begin{abstract}
Information technology has been widely promoted in the healthcare sector to improve current practice and patient safety. However, end users are seldom involved extensively in the design and development of healthcare systems, with lip service often paid to the idea of true user involvement. In this case study the impact of sustained authentic user participation was explored using an interdisciplinary team, consisting of experts both in interaction and healthcare design and consultant anaesthetists, nurses, and pharmacists, to create an electronic prescribing and administration system. This paper details the interface that was created and provides examples of the way in which the design evolved in response to the sustained authentic user participation methods. The working prototype both reduced the opportunity for user error and was preferred by its users to the existing manual system.
\end{abstract}

Keywords: Healthcare, user engagement, participatory design.

\section{Introduction}

The appropriate use of information technology has been promoted in the healthcare sector, both in the UK and abroad, to improve current practice and patient safety. The UK National Programme for IT in the NHS has been widely criticised for running behind schedule and producing solutions that have not always been deemed as satisfactory [1]. Failure to engage with clinicians during the initial stages of the design has been cited as a reason.

This study was based in a hospital setting and created a prototype to allow optimum engagement of users from multiple disciplines in the design and development of an interactive healthcare system. The aim was to design an interface to actively reduce errors, be highly usable and not negatively affect patient care.

\section{Background}

\subsection{The Incidence of Hospital Medication Errors}

\subsubsection{Medication Errors}

The Department of Health [2] estimates that one in 10 patients admitted to NHS hospitals will be unintentionally harmed, with medication errors being the second highest reported cause of harm. 
Medication errors are a global problem [3,4]. It is estimated that between 44,000 98,000 deaths per year are attributable to medication errors in the United States of America [5] with an unknown number in the UK, due to low reporting rates [2].

Medication errors adversely affect the patient, their family and friends, and the professionals concerned. As well as the human cost, they also impose a financial burden on the NHS. The Department of Health estimated that the direct cost of medication errors in 2004 was $£ 200-£ 400$ million per year [6].

\subsubsection{Prescribing Errors}

The growth in the number of available medicines over recent years makes it increasingly difficult for prescribers to be alert to all potential medication interactions. Consequently prescribing has become very complex. When looking at the types of medication error it is believed that prescribing errors are not only the most likely to cause an adverse drug event but are also on the whole avoidable [7].

Common factors associated with prescribing errors have been reported [4] as:

- Decline in renal or hepatic function requiring alteration of drug therapy $(13.9 \%)$

- Patient history of allergy to the same medication class $(12.1 \%)$

- Using the wrong drug name, dosage form or abbreviation $(11.4 \%)$

- Incorrect dosage calculation $(11.1 \%)$

- Unusual and critical dosage frequency considerations (10.8\%).

Approximately 200 million prescriptions are generated in NHS hospitals each year [6]. Almost all are handwritten. A study assessing the quality of handwritten prescriptions found that out of 4,536 inpatient prescriptions, 4-10\% were illegible or ambiguous [8], which can result in wrong drugs or dosages being given. The positioning of zeros and decimal points also give rise to errors. Trailing zeros are sometimes used, for example $1.0 \mathrm{mg}$, intended to mean $1 \mathrm{mg}$, may be misread as $10 \mathrm{mg}$ if the decimal point is not seen, resulting in a ten fold overdose. Handwritten prescription sheets are also subject to transcription errors [7]. These occur when a prescription sheet becomes full and current prescriptions are rewritten onto another sheet, increasing the opportunity for human error.

Prescribing and recording the administration of medicines in intensive care is a complex process, involving a number of disciplines working in a stressful clinical environment. Patients in an intensive care unit are frequently prescribed multiple medicines, often in complicated combinations, therefore increasing the possibility of interactions. This complexity increases the risk of medication errors, which may have catastrophic consequences [6]. This would suggest the Intensive Care Unit (ICU) is a good setting [9] for studying the effectiveness of a suitably designed electronic prescribing system.

\subsection{The Manual Prescribing System}

The ICU in Ninewells Hospital, Dundee uses a manual prescribing system comprising

i) A paper medication chart $(\operatorname{Kardex} 囚)$ to record the patients' prescriptions (figure 1)

ii) A separate card (the Medicine Recording sheet) to record drug administration times. 


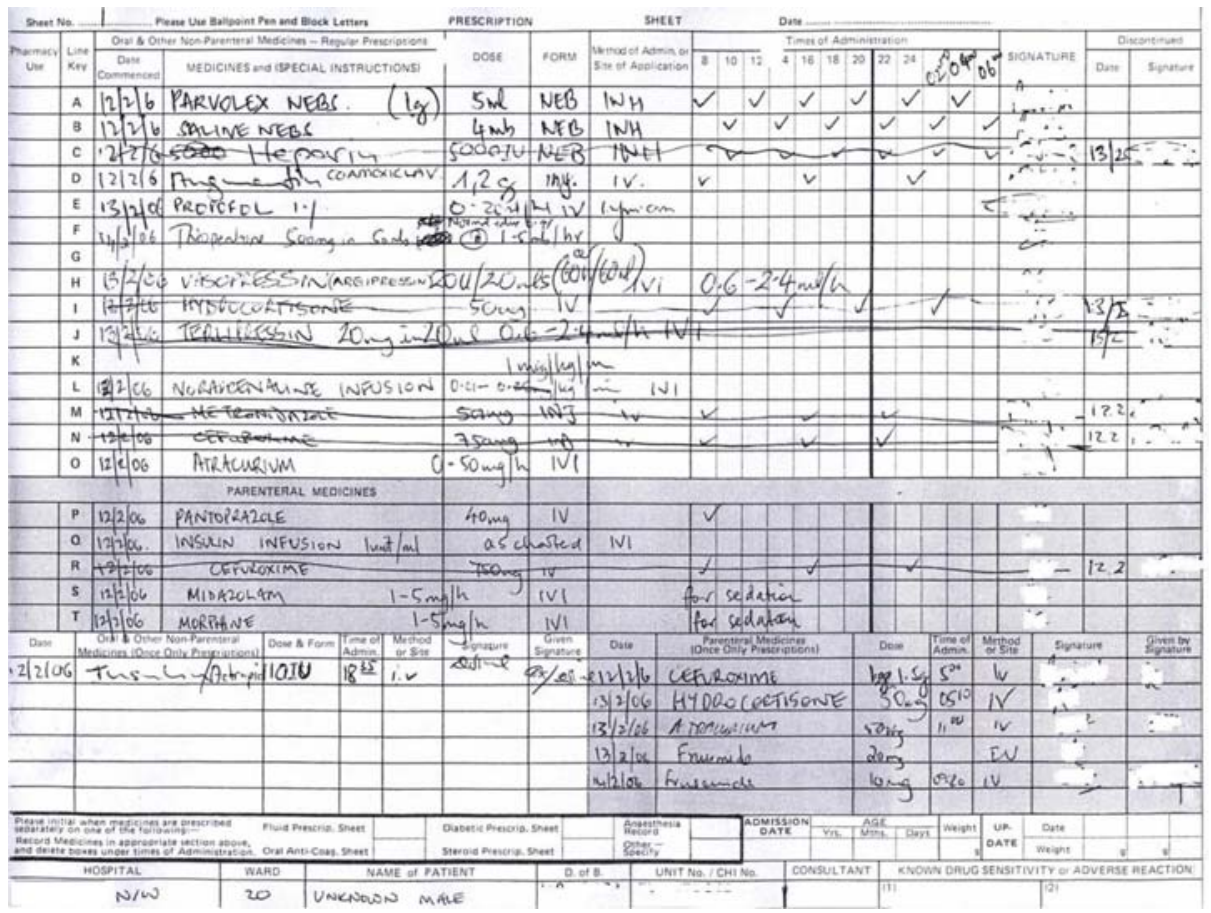

Fig. 1. Patient's Medication Chart from the ICU

\subsubsection{Paper Medication Chart}

The paper medication chart records the start date, name of the drug, dose, route, formulation, time/s of administration and the signature of the prescriber. The details of discontinued prescriptions are also recorded on the chart.

The prescriptions on the chart are separated into two sections. The top section details the regular prescriptions that are required at specified times until discontinued. The lower section details prescriptions that are given only once at the time and date specified.

The chart also contains the patient's personal details recording their name, date of birth, a unique patient identifier used in Scotland (known as the Community Health Index or CHI), consultant, ward, hospital, weight and any allergies.

\subsubsection{Medicine Recording Sheet}

A Medicine Recording sheet is used by the nursing staff to document the times that medications are administered and reasons for non or late administration e.g. "once only medication given" or "dose withheld on prescriber's instruction".

\subsection{Problems Associated with the Manual Prescribing System}

In addition to the problems associated with illegible handwriting, the following system failures are also associated with the paper system: 
- The medication chart and medicine recording sheet fail to provide any backup record.

- If there is damage or spoilage to the chart, it can be difficult to ascertain what medicines a patient has been prescribed or administered, particularly if documentation needs to be reviewed at a later date, for example during the investigation of a complaint or incident.

- There is no mechanism to ensure all appropriate details are provided; for example, a prescription may be discontinued but not clearly crossed out and mistakenly interpreted as a prescription still to be administered.

- Problems can arise when the dosage unit "micrograms" is not written in full, i.e. written as " $\mu \mathrm{g}$ ", which can be misinterpreted as "mg" (milligrams), increasing the likelihood of an overdose being administered.

- If the generic name is not recorded, this can result in the same prescription being entered twice i.e. once with the generic name and a second time using a brand name.

- When the medication chart becomes full, the current prescriptions (i.e. those which have not been discontinued) are transcribed to a new card. There is further potential for errors at this point, in that the information may be transcribed incorrectly as a result of illegible handwriting, human error or incomplete prescriptions.

An electronic system could potentially reduce or eliminate the problems that are prevalent with the paper system and improve patient safety. However, studies in the UK and abroad have identified that such systems can introduce new types of errors [10-15]. Koppel et al [13] discovered 22 types of medication error risks that were facilitated by an electronic prescribing system. Causes of these errors were found to be "human-machine interface flaws" and "information errors". Not only were errors prevalent but they also occurred frequently: this study looked specifically at reducing the opportunity for error by following interaction design practice combined with early and continual authentic user participation.

\section{Designing the System with Authentic User Participation}

The paper medication chart is normally kept in a folder at the end of the patient's bed. The chart is accessed by nurses, doctors and pharmacists and can be viewed by multiple users simultaneously. The electronic system needed to replicate this and also be portable to prevent restricting the normal interaction process that was observed with the paper system e.g. nurses taking the paper chart to the doctor if they had a query about a prescription or passing the chart between the team in the morning ward round. This led to the decision to use a tablet pc.

The system was designed as a client server application, allowing several client machines to be connected to the server, providing each patient with a tablet machine, with a tablet pen for input and an incorporated barcode scanner to allow users to log on to the system. The tablet pc could be mounted at the end of the patients' bed to provide easy viewing and be easily removed from the mount. It would be roughly the 
same size as the paper chart. A rugged tablet pc was required to endure the Intensive Care environment, sustaining extensive cleaning and being dropped. A tablet pen was used for input instead of a keyboard for portability and to reduce the risk of infection.

\subsection{Authentic User Participation}

Involving users as a central part of the development process can lead to more effective, efficient and safer systems [16]. A user-involved approach was adopted for the design, development and implementation of the electronic prescribing and administration system.

At the beginning of the project an interdisciplinary team was formed comprising two consultants in anaesthesia and intensive care, the principal clinical pharmacist for critical care, the intensive care specialist liaison nurse, a nursing education specialist, a professor of interactive systems design, a professor of assistive systems and healthcare computing and a $\mathrm{PhD}$ computing student. The established team was authentically involved throughout the design, development and implementation of the system and met 48 times over a three-year period. The frequency of the meetings ranged between fortnightly and monthly.

A participatory design approach was central to the process. Evolutionary prototyping, a participatory design method where the "system is delivered to the end users as a series of iterative prototypes, each of which gradually adds functionality" [17], was employed. The design began as a set of paper-based prototypes that quickly enabled a visualisation of the system providing a basis for agreeing the requirements. These paper prototypes led to the development of an evolutionary software prototype.

To sustain engagement and enthusiasm, regular meetings were held at which the evolving prototype was evaluated by the interdisciplinary team. The typical structure of these meetings was a walk-through of the prototype, with particular emphasis on the amendments made since the last meeting, followed by a managed discussion with the team that identified improvements and agreed recommendations for changes to the interface, to support best practice. Adopting this process authentically ensured that the evolving system focused on the clinicians' requirements.

\subsection{Interface Design}

The interface of the system was designed by taking cognisance of best practice in interaction design in combination with authentic user participation.

The final system (figure 2) was designed to be familiar and emulate the original paper medication chart with the additional functionality of confirming that medication had been administered. The main functionality of the system was that it allowed

- Doctors to create and discontinue prescriptions

- Nurses to record the administration of medication

- Pharmacists to record that they had checked a prescription and add additional notes to the prescription

- New patients to be added to the system

- Authorised users to amend patient details 


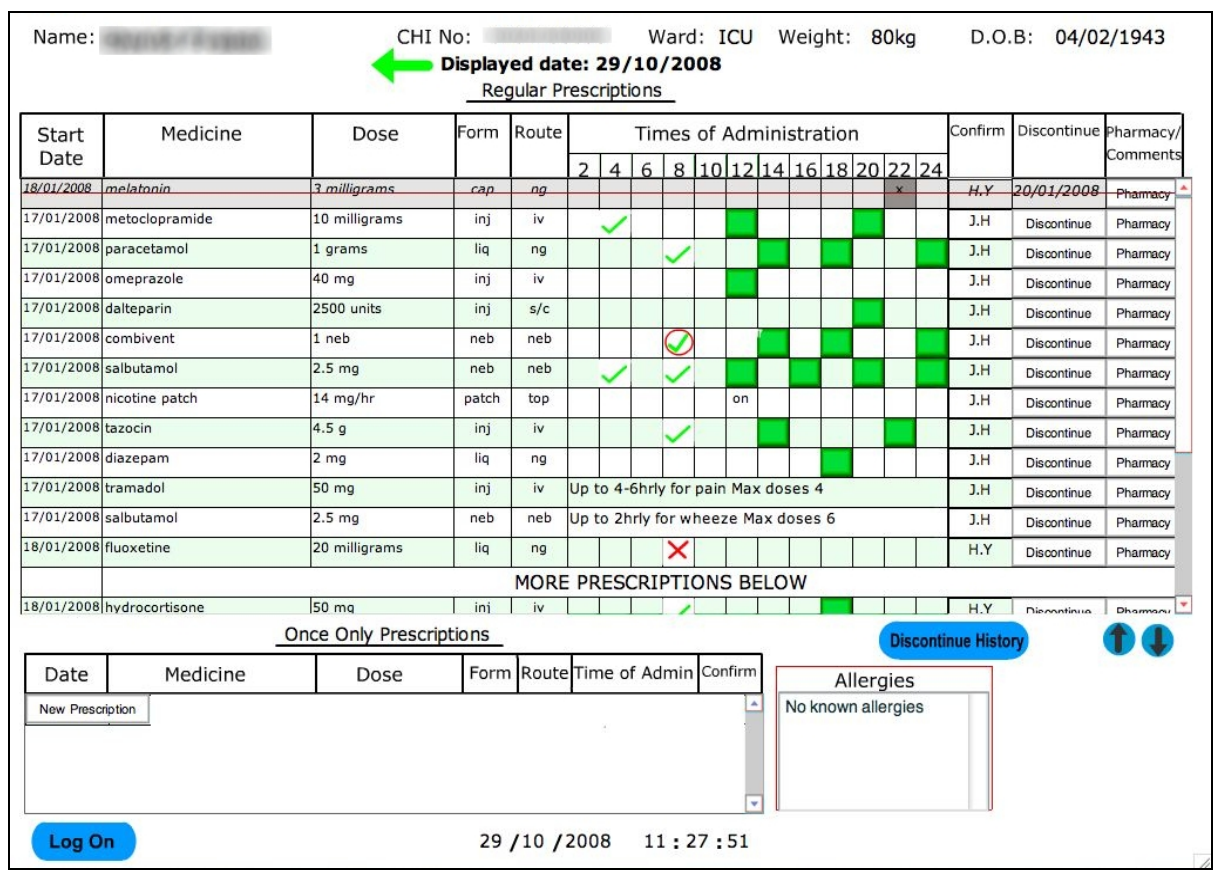

Fig. 2. The Final System

The interface was organized into two sections, similar to the paper system, with the top section displaying regular prescriptions and the bottom section displaying the "once only" prescriptions. Within the regular prescription section three subsections displayed discontinued, current and future prescriptions.

Within the "regular prescriptions" continuous infusions were grouped together as a consequence of nursing staff feedback, to make the administration process easier and to reflect the sequence for administering medication. The interface was designed to minimise free text input, to avoid frustration and save time entering text via an onscreen keyboard.

\subsection{Decision Support}

Some commonly prescribed medications such as insulin and gentamicin have associated algorithms to determine the amount and frequency of doses given to the patient, based for example on patient weight. Patients in intensive care often behave as if they are diabetic. It has been found that a lower target range than normally aimed for of blood glucose in these patients can improve their outcome [18,19].

It was initially decided that because insulin was frequently prescribed in ICU, it would be beneficial to incorporate an insulin-prescribing algorithm [20] into the system.

The clinicians sourced and evaluated an algorithm, which the team further refined during the course of eleven meetings, so that it could be successfully incorporated into the evolutionary prototype. 
There is also a high prevalence of renal and hepatic impairments in ICU patients, requiring the usual medication dose to be altered. When creating a new prescription, the team decided to display relevant dose recommendations, as specified by the British National Formulary (BNF), after a medication had been selected.

Both the insulin prescribing algorithm and the medication dose recommendations are examples of decision support. During the course of numerous meetings, the advantages and disadvantages of incorporating decision support were debated. It was highlighted that the dose recommendations included in the system conflicted with other guidelines in use in the ICU. After careful consideration by the team, it was decided that implementing decision support, in a piecemeal fashion, could introduce errors and cause annoyance to users by providing inconsistent information. Furthermore, it could introduce an additional variable, which could impact on the comparison of the paper and electronic systems. Therefore, it was proposed to concentrate on creating an interface that minimised prescribing errors and to evaluate its effectiveness before implementing decision support. The team agreed to remove the insulin prescribing algorithm and any medication impairment recommendations.

\subsection{Wish List}

Throughout the process, requirements were identified that the team believed would be useful (e.g. incorporating the 24-hour chart functionality) but would not directly affect the level of prescribing errors or the usability. As the purpose was to design a usable system to reduce prescribing errors, it was decided by the team that these requirements could be implemented at a later date and would be part of an "ideal" system. A wish list was suggested by one of the clinicians to prevent these ideas from being forgotten and to also lessen the risk of demotivation, which could lead to a reduction in contributions from group members.

\subsection{New Prescription Design}

Following one of the evaluation sessions, it became evident that multiple selections were needed to create a new prescription and that this could be avoided by using a software "wizard" for the task. In addition to reducing the number of screen selections, this would ensure that the users would adopt the correct order for creating a new prescription, by breaking down the process into the logical order for construction of a prescription.

Creating a prescription with the original prototype involved selecting the start date, medicine, times of administration, and confirm and selecting options from the route and form drop down menus, all of which were located underneath the last completed prescription line as shown in figure 3 .

\begin{tabular}{|c|c|c|c|c|c|c|c|c|c|}
\hline $14 / 04 / 20 n 5$ & heparin & 750 unit/ml & inj & & inf & As clotting time & $B . T$ & $12 / 4$ & Phomacy \\
\hline Start Date & Medicine & $\mathbf{v}$ & inj & $\nabla i$ & inf & Times of Administration & Confirm & Discontinue & Phamacy \\
\hline
\end{tabular}

Fig. 3. Initial "New Prescription" Design 


\begin{tabular}{|l|l|l|l|l|l|l|l|l|l|l|l|l|l|l|l|}
\hline $25 / 02 / 2008$ & metoclopramide & 10 milligrams & inj & iv & & $\mathrm{x}$ & & & $\mathrm{x}$ & & & $\mathrm{x}$ & & & $\mathrm{x}$ \\
\hline New Prescription
\end{tabular}

Fig. 4. Revised "New Prescription" Design

The revised "new prescription" design allowed the user to initially select the new prescription (figure 4). The location of the new prescription was retained as the eye was naturally drawn to the point under the last completed prescription.

After selecting the new prescription, the prescription "wizard" was displayed in the middle of the screen to attract the users attention.

- Firstly a calendar was displayed prompting the user to enter a start date.

- Next, the screen refreshed to prompt the user to select a medicine. There are over 100 commonly prescribed medicines in ICU, which led to a discussion on how best to display the different medicines. This resulted in the decision to use a filing card layout to allow the user to easily select the required medicine. They would select which letter range the medicine was in and then select the appropriate medicine from a list of options. This screen also displayed the start date selected and a back arrow providing the opportunity to return to the previous screen.

- To avoid duplicate prescriptions, if the medicine was currently prescribed, a warning was displayed to the prescriber presenting two options to either continue creating the prescription or cancel creating the new prescription.

- If they selected "continue" or if there were no duplicate prescriptions, a screen prompting the user to select the appropriate route of the medicine was presented, followed by a screen to prompt selection of the appropriate formulation of the medicine.

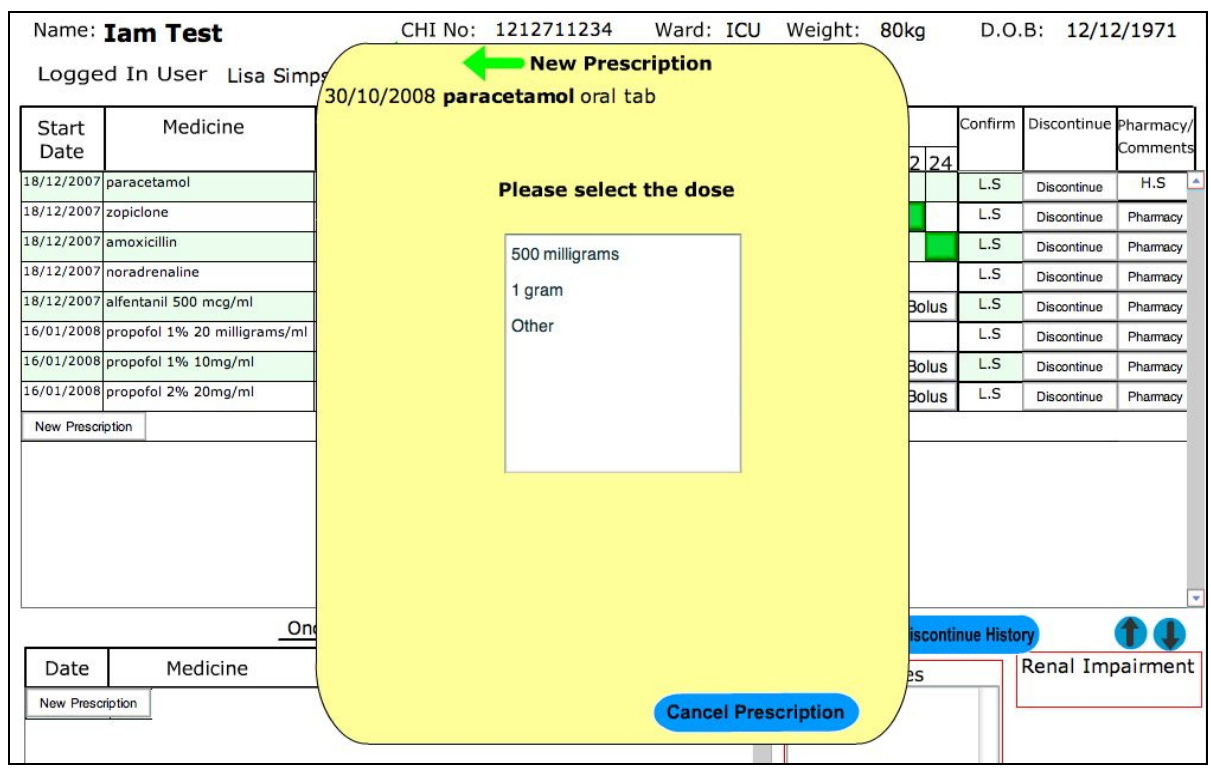

Fig. 5. New Prescription "Wizard" 
- Based on these selections, the next screen displayed the appropriate dose in addition to the selected start date, medicine, route and form (figure 5).

- This was then followed with a screen prompting the user to select the times of administration and then a screen to confirm the prescription.

\subsection{Minimising Error}

The prescription wizard further reduced opportunities for errors. From a survey of prescription errors carried out in the ICU [21], the omission of important information occurred in over a quarter of the non-compliant prescriptions e.g. missing dose. The prescription wizard was developed to not only lessen the number of screen selections but to minimise the risk of important omissions.

In addition, the wizard was also designed to reduce the likelihood of entering inappropriate or incomplete information by providing suitable formulations, route and dose defaults, appropriate to the selected medicine. Once the medication was selected, the screen displaying the appropriate route options would be populated based on the medication. Subsequent to the route selection, the screen would then refresh to display the available formulations resulting from previous selections of medication and route. Underneath any default lists an "other" option was always provided to maintain flexibility and allow the user to prescribe a non-routine selection. This error prevention mechanism of displaying dynamic default options not only reduced the number of physical actions necessary to input the required prescription information but also assisted the prescriber by recognition.

However, although displaying a list of default options can reduce the likelihood of users entering inappropriate and incomplete information it can introduce human error via "slips" [22] if an unintended option is selected. For example Shulman et al [14] reported that one of the major errors caused by an electronic prescribing system occurred when the wrong dose was selected from a pull down menu and could have resulted in a 70 times overdose To minimise this, when an option was selected via the "wizard" it was again displayed in the next window with an option to return to the previous screen if an amendment is required. Once all the required prescription details had been selected, the created prescription was then displayed in a different format for the user to check and confirm, before the prescription was finalised. This double check mechanism was incorporated into the wizard to reduce the likelihood of erroneous selections, which could cause serious patient harm.

\section{Evaluation Results}

Expert analysis and user participation methods [23] were used to evaluate the evolutionary prototype electronic prescribing and administration system. The expert analysis method comprised three heuristic evaluations conducted at regular intervals throughout the development of the prototype to evaluate whether the system adhered to accepted usability principles [24]. In addition to the evaluation sessions held with the clinicians in the interdisciplinary team, end users outwith the team evaluated the system in both a laboratory and clinical setting where observational methods (cooperative evaluation) and query techniques (user interviews) were used respectively. 
Subsequent to the team decision that the prototype of the electronic system appeared to meet the necessary requirements of the different user groups, two evaluation sessions with 30 clinicians were held; one with prescribers and one with administrators.

Following 59 evaluation sessions (including the 48 held with the interdisciplinary team) the prototype electronic prescribing and administration system was evaluated using live prescription information in the ICU. The prototype was used with 16 patients over a 5 -month period $\left(14^{\text {th }}\right.$ January $-5^{\text {th }}$ June 2008$)$. Paper and electronic prescription charts were run in parallel within ICU.

\subsection{Levels of Prescribing Error}

On completion of the 5-month trial both the electronic and paper medication charts were surveyed for levels of prescribing errors. The results of the study demonstrated that the overall level of prescription compliance was significantly higher with the electronic system (91.67\%) compared to the paper system (46.73\%). Electronically generated prescriptions were unambiguous, legible and resulted in a significant reduction in identified prescribing deviations.

However, there was a decrease in the level of compliance for infusions created on the electronic system, where both the rate and concentration of the medication was not always stated. This was due to a flaw in the new infusion prescription design and the integrity of data used to populate the drug database. Evaluating the system in parallel with the paper chart allowed this flaw to be identified without any risk to the level of patient care.

\subsection{Interviews}

From a total population of 62 clinicians who used the system, a sample was interviewed, comprising 9 doctors, 10 nurses and a clinical pharmacist. Nurses were the most frequent users of the system. Interviews took place between $28^{\text {th }}$ April $-23^{\text {rd }}$ July 2008. Participants had different levels of use of the system and only one participant had previous experience of electronic prescribing.

Overall the electronic system was preferred (60\% of participants, 6 doctors, 6 nurses). Examples of the comments from the staff are shown in box 1.

The users' experience was on the whole positive. Generally they found the system intuitive, easy to learn and they were comfortable using it (box 2).

None of the participants interviewed expressed discomfort with the system. Five of the participants expressed initial reservations, with three of the five expressing a change of impression to a positive reaction after using the system.

"Anything that's new you think it's going to go wrong and because it is prescribing as well, prescribing is important to be right... I suppose there was a concern that it might cause a problem with prescribing" When asked if this impression changed over time "Yes, I like your system!" (Specialist registrar)

The other 2 participants changed to expressing an acceptance for the system but did not express a liking.

Eleven of the twenty participants said they believed the electronic prescribing and administration system had a positive impact on patient safety as it "reduce (d) errors" and was "a safer system to use". Although the remaining participants did not explicitly say that the electronic system had a positive impact they implied that it would improve patient safety and would not have a negative effect. 
Box 1. Comments from the staff about the electronic system

\section{Examples of preference for using the system}

"I found it easier to go through the electronic one and see what had been given throughout the day instead of flicking through pages and pages of paper. It's a lot clearer and easier to decipher the information" (Staff nurse)

"I liked it, it's good, it's concise, it's a joy to be able to read a prescription clearly, understand what it says, the units it's supposed to be in and who has prescribed it. Yeah I really liked it" (Staff nurse)

"I probably prefer for ease of use the electronic one" (Specialist registrar)

"One thing is it's definitely safe, ... Patient safety - I think it's immensely improved and the other thing is it's very legible... and everybody can understand what's happening very clearly" (Specialist registrar)

\section{Example of hesitance towards the electronic system}

"I think probably I prefer the paper because of practice and what I'm used to, but I think if we didn't have to do the paper as well I wouldn't have a problem with doing the electronic" (Staff nurse)

"Writing does seem quicker but I can see that there is probably less room for errors if you do it electronically. They were quite similar really you put the same details in (for both systems). (I preferred using the) Paper, I think it's just getting used to something ... I only had a short period on it" (Specialist registrar)

Box 2. Examples of Users' Experience of the Interaction

"Ideas were really good, the format of the (electronic) Kardex® was good and after a small amount of training you could use the basics. I thought it was quite easy really, to use" (Charge nurse)

"I would say very comfortable it didn't take too long to get the hang of it... It was good, it was simple to use, it was a very intuitive set of interfaces, it was very clear"(Specialist registrar)

"It was quite straightforward actually. Once I got over the shock of an electronic system .... it's actually very straightforward" (Staff nurse)

"Very user friendly...(The layout) mirrored a normal prescription so that's good. The thing that I like about it were that it keeps your allergies, renal impairments, pregnant, hepatic impairment to one bit... I think it worked well" (Specialist registrar) 


\section{Discussion}

The study clearly demonstrates that the participatory approach produced a prototype which reduced prescribing errors by almost doubling levels of prescription compliance and importantly, was accepted by the end users: the doctors, pharmacists and nurses.

This paper illustrates the benefit of sustained user engagement in the design process. The inclusion of the users throughout the design and development process generated an evolving understanding of the process and encouraged ownership of the project.

Although the design decisions made were not necessarily novel, the process taken to reach these decisions highlights the benefits of ongoing participatory user involvement. Sustained engagement allowed the members of an interdisciplinary team to share their ideas, work through a number of options and refinements and delivered a solution that was designed both with and for the users, to meet their various needs. The participants understanding of the process was evidenced by the agreement of the group to create a wish list and remove decision support.

Authentic user participation shaped a system preferred overall by the clinicians to complete their everyday tasks and demonstrated a significant reduction in prescribing errors.

Acknowledgments. We wish to express our gratitude to the clinicians on the development team from the ICU, Ninewells Hospital, Dundee, who made this study possible by giving so generously of both their expertise and their time: P Antoniewicz, DA Corner, S Dailly, J Joss, F McIntyre, S McLeod and AJ Shearer. We also want to extend our thanks to all the clinicians who evaluated the prototypes.

\section{References}

1. Cross, M.: Benefits of $£ 12$ bn IT Programme in NHS are "unclear," MPs Say. BMJ 334, 815 (2007)

2. Department of Health.: A Safer Place for Patients: Learning to Improve Patient Safety. The Stationery Office, London (2005)

3. Bates, D.W., Cullen, D.J., Laird, N., et al.: Incidence of Adverse Drug Events and Potential Adverse Drug Events: Implications for Prevention. JAMA 275, 29-34 (1995)

4. Lesar, T.S., Briceland, L., Stein, D.S.: Factors Related to Errors in Medication Prescribing. JAMA 277, 312-317 (1997)

5. Institute of Medicine: To Err is Human: Building a Safer Health System. National Academic Press, Washington DC (2000)

6. Department of Health: Building a Safer NHS for Patients: Improving Medication Safety. The Stationery Office, London (2004)

7. Wheeler, S.J., Wheeler, D.W.: Medication Errors in Anaesthesia. Anaesthesia 60, 257-273 (2005)

8. Jenkins, D., Cairns, C., Barber, N.: The Quality of Written Inpatient Prescriptions. Int. J. Pharm. Pract. 2, 176-179 (1993)

9. Barber, N.: Designing Information Technology to Support Prescribing Decision Making. Qual. Saf. Health Care 13, 450-454 (2004) 
10. Ash, J.S., Berg, M., Coiera, E.: Some Unintended Consequences of Information Technology in Health Care: The Nature of Patient Care Information System-related Errors. J. Am. Inform. Assoc. 11, 104-112 (2004)

11. Campbell, E.M., Sittig, D.F., Ash, J.A., et al.: Types of Unintended Consequences Related to Computerized Provider Order Entry. J. Am. Inform. Assoc. 13, 547-556 (2006)

12. Donyai, P., O'Grady, K., Jacklin, A., et al.: The Effects of Electronic Prescribing on the Quality of Prescribing. Br. J. Clin. Pharmacol. 65, 230-237 (2008)

13. Koppel, R., Metlay, J.P., Cohen, A., et al.: Role of Computerized Physician Order Entry Systems in Facilitating Medication Errors. JAMA 293, 1197-1203 (2005)

14. Shulman, R., Singer, M., Goldstone, J., et al.: Medication Errors: A Prospective Cohort Study of Hand-written and Computerised Physician Order Entry in the Intensive Care Unit. Crit Care 9, 516-521 (2005)

15. Walsh, K.E., Adams, W.G., Bauchner, H., et al.: Medication Errors Related to Computerized Order Entry for Children. Pediatrics 118, 1872-1879 (2006)

16. Preece, J., Rogers, Y., Sharp, H.: Interaction Design: Beyond Human-Computer Interaction, 2nd edn. John Wiley \& Sons, New York (2007)

17. Muller, M.J.: Participatory Design: The Third Space in HCI. In: Jacko, J.A., Sears, A. (eds.) The Human-Computer Interaction Handbook: Fundamentals, Evolving Technologies and Emerging Applications. Human Factors and Ergonomics, pp. 1051-1068. Lawrence Erlbaum Associates, Hillsdale (2003)

18. Finney, S.J., Zekveld, C., Elia, A., et al.: Glucose Control and Mortality in Critically Ill Patients. JAMA 290, 2041-2047 (2003)

19. Van den Berghe, G., Wouters, P., Weekers, F., et al.: Intensive Insulin Therapy in Critically Ill Patients. N. Engl. J. Med. 345, 1359-1367 (2001)

20. Thomas, A.N., Marchant, A.E., Ogden, M.C., et al.: Implementation of a Tight Glycaemic Control Protocol using a Web-based Insulin Dose Calculator. Anaesthesia 60, 1093-1100 (2005)

21. Went, K., Antoniewicz, P., Corner, D.A., et al.: A Survey of Prescribing Errors in Intensive Care. Scot. Med. J. 52, 50 (2007)

22. Reason, J.: Human Error. Cambridge University Press, Cambridge (1990)

23. Dix, A., Finlay, J., Abowd, G.D., et al.: Human-Computer Interaction, 3rd edn. Pearson Education Ltd., London (2004)

24. Nielsen, J.: Heuristic Evaluation. In: Nielsen, J., Mack, R.L. (eds.) Usability Inspection Methods, pp. 25-62. John Wiley, New York (1994) 